\title{
On Proper Phase Contrast Imaging in Aberration Corrected TEM
}

\author{
Peter Hartel, Martin Linck, Frank Kahl, Heiko Müller and Max. Haider \\ Corrected Electron Optical Systems GmbH, Heidelberg, Germany
}

Since the realization of the first aberration corrected TEM [1] the number of aberration corrected TEMs is still rapidly increasing. Two key benefits have enabled this tremendous success of spherical aberration correction: improved point resolution limit and vanishing delocalization. Many thoughts have been spent on the proper 'design' of the phase contrast transfer function (PCTF) of an 'ideal' microscope, where only round aberration coefficients (defocus $\mathrm{C} 1$ and spherical aberration coefficients of different orders $(\mathrm{Cs}=\mathrm{C} 3, \mathrm{C} 5, \ldots)$ are taken into account, see e.g. [2, 3]. In practice, however, many users only pay attention to a (global) $\pi / 4$-limit for all or part of the aberration coefficients. Here we want to bridge the gap between the two approaches and point the way to achieve proper phase contrast imaging even if unavoidable residual aberrations are present.

As a first step away from a global $\pi / 4$-limit, the contributions of the round aberrations (PCTF!) and nonround aberrations have to be considered separately: While for the latter a global $\pi / 4$-limit might be useful, the round aberrations have to create a broad transfer band close to $+\pi / 2$ or $-\pi / 2$ for optimum phase contrast - which is clearly not in line with a $\pi / 4$-criterion.

Secondly, single-coefficient $\pi / 4$-limits are problematic for two reasons: (I) The $\pi / 4$-limit does not encounter for the specific shape of the phase shift over the entire aperture. This is known from light optics [4]. Criteria, which optimize e.g. the integrated mean quadratic distance $\left(\mathrm{D}_{\mathrm{ph}}\right)$ from the ideal phase over the effective aperture, are more realistic. Fig. 1 shows the phase shifts generated by all axial aberration coefficients up to fifth order. For the same $\pi / 4$-limit, $D_{p h}$ of the two-fold astigmatism $A_{1}$ is by a factor of two smaller than $D_{p h}$ for the defocus $C_{1}$ due to the different shape of the phase plates (zerolines for $\mathrm{A}_{1}$ ). (II) Compensation schemes can be applied for aberrations of same multiplicity but different orders. In Fig. 1 potential partners are arranged within one column. The value of the highestorder coefficient and the size of the effective aperture (= maximum transferable spatial frequency) determine the optimum values for the lower-order coefficients (e.g. multiplicity 2: $\mathrm{S}_{5}$ given; $\mathrm{S}_{3}, \mathrm{~A}_{1}$ optimized). Hereby $\mathrm{D}_{\mathrm{ph}}$ for each multiplicity is minimized (ideal phase of zero). This procedure is similar to adjusting $\mathrm{C}_{3}$ and $\mathrm{C}_{1}$ optimum for a given $\mathrm{C}_{5}-$ except that the ideal phase is $+\pi / 2$ or $-\pi / 2$ for the PCTF.

In Fig. 2 an example of the SALVE II microscope [5] at 40kV is given. The phase plate (a) corresponds to the output of the CEOS software after aberration correction. The $\pi / 4$-circle is misleadingly small as it is largely determined by $\mathrm{C}_{3}$. In image (b) all round aberrations (PCTF) are omitted. This gives a more realistic picture of the residual aberrations. In (c) an optimum $C_{1}$ for given $C_{3}$ and $C_{5}$ is chosen and the resulting passband is visualized. With a full compensation scheme $(\mathrm{d}$, e) for fourth-and fifth-order aberrations by means of all lower order aberrations, a passband of up to $50 \mathrm{mrad}$ can be achieved.

\section{References:}

[1] M. Haider et al, Nature 392 (1998), 768-769.

[2] O. Scherzer, Ber. Bunsen-Gesellschaft phys. Chemie 74 (1970), 1154-1167. 
[3] M. Lentzen, Microsc. Microanal. 14 (2008), 16-26.

[4] M. Born, E. Wolf, Principles of Optics, $6^{\text {th }}$ edition (Cambridge university press, Cambridge), p. 468.

[5] SALVE II project, <http://www.salve-project.de>.

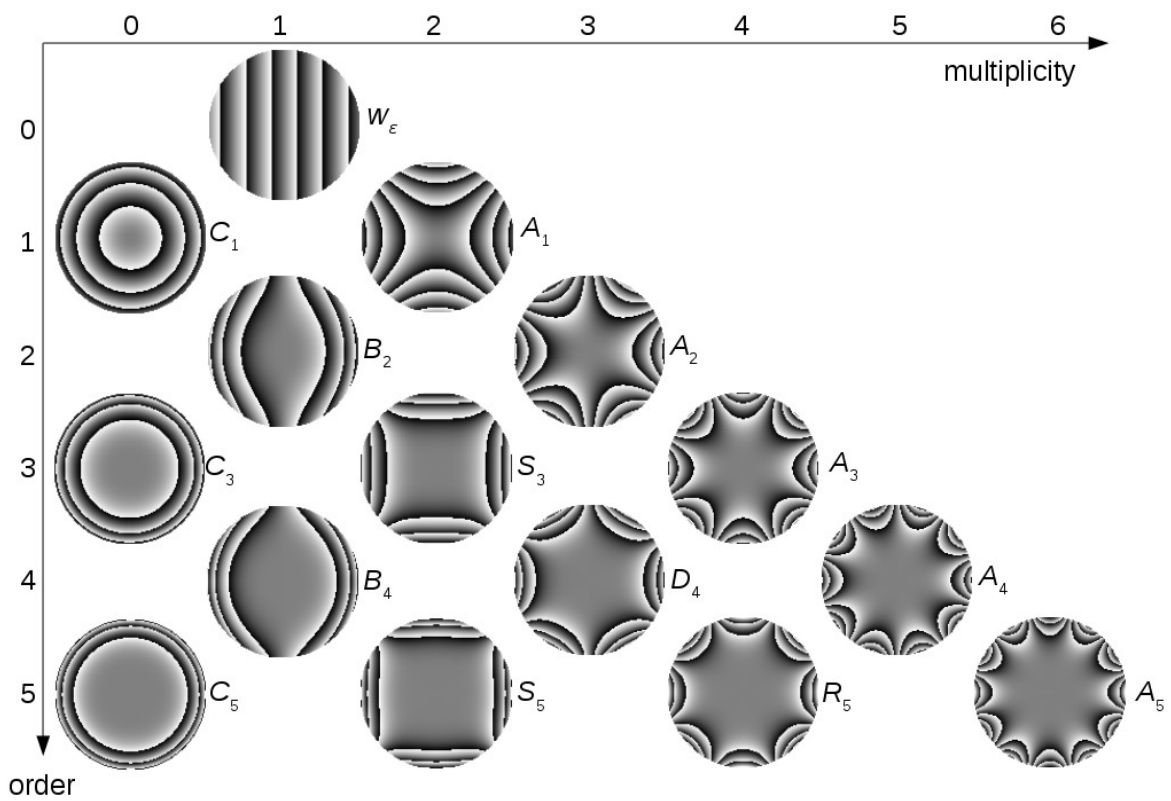

Figure 1. Table of axial aberrations visualized as phase plates. At the aperture edge $\pm 6 \pi$ is adopted; the phase is wrapped to $[-\pi$ (black); $\pi$ (white)[. Aberrations of same multiplicity but different order can partly compensate each other. The round aberrations create the PCTF.
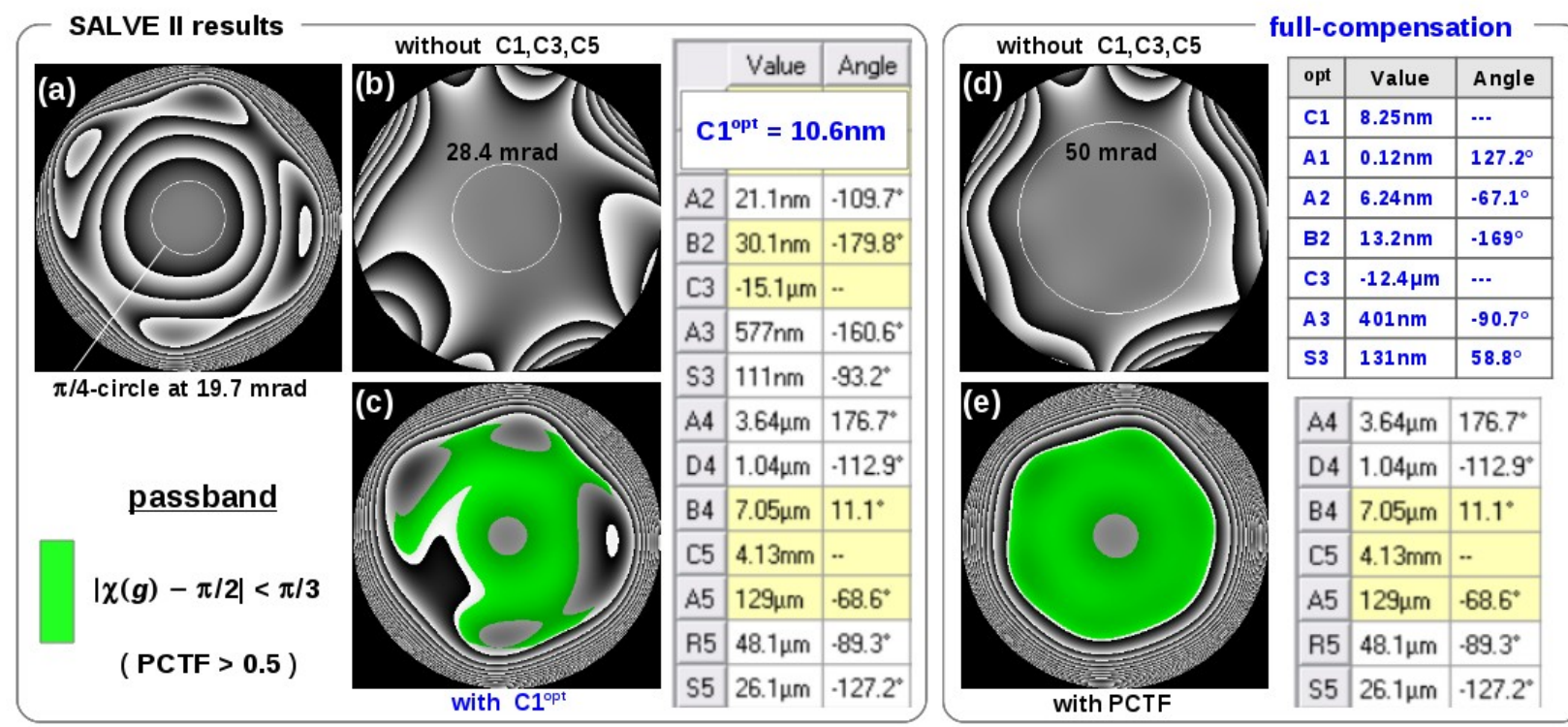

\begin{tabular}{|l|l|l|}
\hline A4 & $3.64 \mu \mathrm{m}$ & $176.7^{\circ}$ \\
\hline D4 & $1.04 \mu \mathrm{m}$ & $-112.9^{\circ}$ \\
\hline B4 & $7.05 \mu \mathrm{m}$ & $11.1^{\circ}$ \\
\hline C5 & $4.13 \mathrm{~mm}$ & - \\
\hline A5 & $129 \mu \mathrm{m}$ & $-68.6^{\circ}$ \\
\hline R5 & $48.1 \mu \mathrm{m}$ & $-89.3^{\circ}$ \\
\hline S5 & $26.1 \mu \mathrm{m}$ & $-127.2^{\circ}$ \\
\hline
\end{tabular}

Figure 2. Left: Phase plates for measured aberration coefficients at the SALVE II microscope operated at $40 \mathrm{kV}$. The passband in (c) demonstrates the reduced contrast transfer due to residual aberrations. Right: With a suitable compensation scheme a passband up to $50 \mathrm{mrad}$ can be achieved. The aperture radius of all phase plates amounts to $75 \mathrm{mrad}$. 